Psychotherapeut 2019 64:186-193

https://doi.org/10.1007/s00278-019-0351-3

Online publiziert: 6. Mai 2019

(c) Der/die Autor(en) 2019

\section{Redaktion}

Anja Hilbert, Leipzig

Ann-Christine Ehlis, Tübingen

Stefanie Enriquez-Geppert ${ }^{1,2}$

${ }^{1}$ Abteilung für Klinische und Entwicklungs-Neuropsychologie, Universität Groningen, Groningen, Niederlande

${ }^{2}$ Abteilung für Biomedizinische Wissenschaften, Sektion Kognitive Neuropsychiatrie, Universitätsklinikum Groningen, Groningen, Niederlande

\title{
Neurofeedback aus der Perspektive der Neurowissenschaften
}

\section{Aktuelle Entwicklungen und Trends}

\begin{abstract}
Neurofeedback (NF) weckt derzeit erhöhtes Interesse vonseiten der Wissenschaft und klinischen Anwendung. Dies liegt v. a. an zwei Trends: dem Technikfortschritt sowie dem Zuwachs des Wissens über Zusammenhänge von neuronalen Funktionen und pathologischer Manifestation. Letzteres ermöglicht die Entwicklung neuer Protokolle für die klinische Praxis im Sinne einer individualisierten, am klinischen Bedarf orientierten Therapie. Nur wenn es in der Forschung zum verstärkten Einsatz von methodisch hochwertigen Studien kommt, kann die klinische Anwendung zukünftig von diesen Entwicklungen profitieren.
\end{abstract}

\section{Definition, Mechanismen und Einsatzbereiche der Neurofeedbackmethode}

Es sieht zunächst wie ein futuristisches Computerspiel aus: Ohne Joystick oder Tastatur sitzen Personen vor einem Bildschirm und bewegen Raketen oder Autos. Bei genauerer Betrachtung stellt sich heraus, dass das Computerspiel Teil einer Therapie ist, dem sog. Neurofeedback. Auf den Köpfen der Patienten sind Elektroden mithilfe einer Kappe angebracht, die Gehirnströme messen. Bestimmte Aspekte der Gehirnaktivität werden in Echtzeit analysiert und dem Patienten rückgemeldet. Dieses Feedback in Form von Figuren auf dem Bildschirm hilft, die sonst nicht direkt wahrnehmbare und steuerbare Gehirnaktivität zugänglich $\mathrm{zu}$ machen. Je nachdem, ob die aktuelle Gehirnaktivität erwünscht ist oder nicht, bewegen sich die Figuren auf dem Bildschirm weiter oder erstarren. Dadurch erlernt der Patient mentale Strategien und setzt sie ein, um neuronale Merkmale zu regulieren, die mit bestimmten kognitiven Funktionen oder der Reduzierung von Symptomen zusammenhängen (•Infobox 1$)$.

Neurofeedback informiert den Patienten durchgehend über seine aktuelle Gehirnaktivität, damit er bestimmte neuronale Aspekte selbst regulieren lernt. Die hierfür benötigte Gehirn-Computer-Schnittstelle wird durch eine Rückmeldeschleife mit 5 Elementen gebildet (-Abb. 1):

1. Messung der Gehirnaktivität durch bildgebende Verfahren wie Elektroenzephalographie (EEG), Magnetenzephalographie, Magnetresonanztomographie oder Nahinfrarotspektroskopie;

\footnotetext{
Infobox 1 Empfohlene Literatur I

- Strehl S (Hrsg) (2013) Neurofeedback. Theoretische Grundlagen, praktisches Vorgehen, wissenschaftliche Evidenz. Kohlhammer, Stuttgart

- Haus K-M, Held C, Kowalski A et al (Hrsg) (2016) Praxisbuch Biofeedback und Neurofeedback. Springer, Berlin Heidelberg New York Tokio
}

2. Echtzeitanalyse der gemessenen Aktivität (einschließlich Herausfiltern von Messartefakten);

3. Extraktion eines bestimmten neuronalen Merkmals, das trainiert werden soll (z. B. ein bestimmtes Frequenzband);

4. Übertragung dieses Merkmals in ein Feedbacksignal sowie

5. der Patient, der versucht seine Gehirnaktivität zu beeinflussen, die dann wiederum gemessen, analysiert, extrahiert und rückgemeldet wird.

Merke. Obwohl NF potenziell mit unterschiedlichen Methoden durchgeführt werden kann und andere Modalitäten als EEG vielversprechende Resultate zeigen, hat derzeit die EEG die größte Bedeutung in der klinischen Praxis: Neurofeedback-

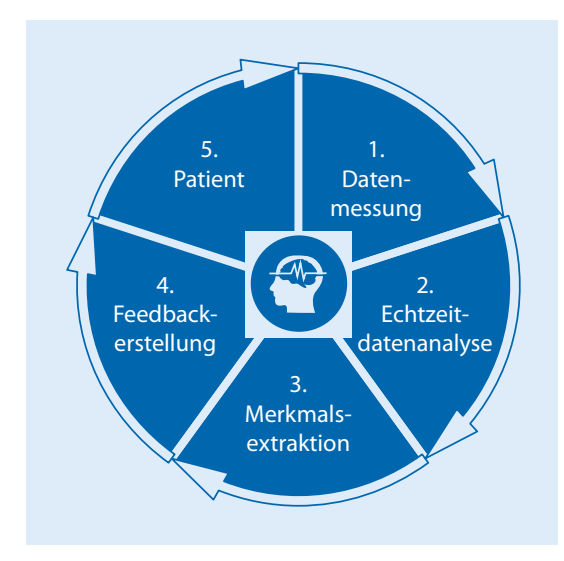

Abb. 1 \ Fünf Elemente der Neurofeedback-Rückmeldeschleife 


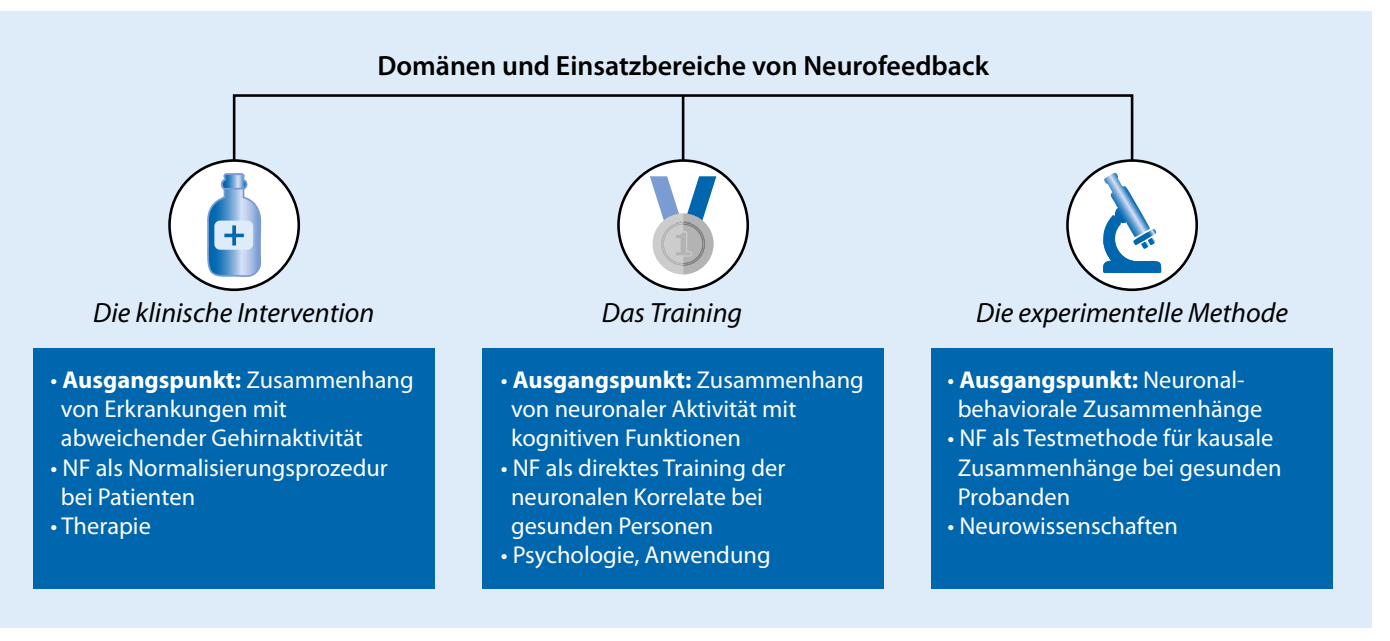

Abb. $2 \triangleleft$ Einsatzbereiche des Neurofeedbacks (NF)

Software und -Hardware sind mobil und kostengünstig.

Die Ausgestaltung dieser Elemente ist Grundlage eines NF-Protokolls. Diese betrifft u.a. die Festlegung auf ein bestimmtes Messverfahren, ein aus einer bestimmten Region extrahiertes, neuronales Aktivitätsmerkmal und die Art des Feedbacks. Unterscheidet sich zwischen sonst identischen Protokollen allein die Auswahl der Elektrodenposition (z.B. frontale vs. parietale Regionen), können dadurch unterschiedliche neuronale Netzwerke angesprochen werden, die unterschiedlichen kognitiven Funktionen unterliegen. Zudem kann sich die Effizienz von Protokollen trotz Auswahl eines gleichen und aus derselben Region extrahierten neuronalen Merkmals unterscheiden, wenn z. B. die Sitzungsanzahl abweichend ist.

Derzeit wird NF hauptsächlich in folgenden 3 Bereichen genutzt: als (i) therapeutisches Verfahren bei Patienten, (ii) Training zur Leistungsoptimierung gesunder Personen, (iii) experimentelle Methode zur Untersuchung eines möglichen kausalen Zusammenhangs eines neuronalen Merkmals und einer kognitiven Funktion (Enriquez-Geppert et al. 2017; - Abb. 2; - Infobox 2).

Bei NF werden unterschiedliche Lernmechanismen diskutiert. (i) operantes und klassisches Konditionieren gehören $\mathrm{zu}$ den wichtigsten und werden von Ros et al. (2014) basierend auf der NFRückmeldeschleife beschrieben. Frühe NF-Phasen sind zunächst besonders durch fluktuierende Feedback-Signale charakterisiert, die stochastische und unkonditionierte neuronale Variabilität widerspiegeln. Nachfolgend erreicht die neuronale Aktivität sporadisch und zufällig den Bereich der erwünschten Aktivität, bei dem der Proband entsprechendes Feedback erhält. Dadurch ist das Gehirn in der Lage, einen bestimmten neuronalen Zustand als internen Sollwert zu speichern, und schüttet belohnungsmodulierende Signale wie Dopamin aus, die auch für die neuronale Plastizität wichtig sind. Bei nachfolgenden Rückmeldeschleifen versucht der Proband, die erwünschte Gehirnaktivität durch Anwendung mentaler Strategien zu reproduzieren, verwendet idealerweise immer effizientere Strategien, trifft den Sollwert besser und verändert somit leichter seine Gehirnaktivität. (ii) Einen weiteren Mechanismus stellt die ZweiProzess-Theorie von Lacroix (1986) dar, die von Wood et al. (2014) um 3, im NFKontext wesentliche, neuronale Netzwerke erweitert wurde. (iii) Letztendlich spielt auch der Erwerb von Fertigkeiten (hierbei das Erlernen der Selbstregulation der Gehirnaktivität) eine Rolle.

Folgende 3 neuroplastische Mechanismen stehen bei NF im Fokus. (i) Gemäß der Hebb-Plastizität konditioniert NF ein bestimmtes neuronales Aktivitätsmuster. Wenn Neurone vermehrt gemeinsam feuern, werden ihre Verbindungen gestärkt; dies vereinfacht das zukünftige Entstehen desselben Aktivitätsmusters (Ros et al. 2014). (ii) Homöostatische Plastizität bezeichnet einen intrinsischen Regulationsmechanismus, der das
Erreichen von pathologischen Extremzuständen neuronaler Aktivität bei Gesunden verhindert. Wenn dieser Mechanismus beeinträchtigt ist, kann NF ihn normalisieren. Homöostatische Plastizität äußert sich durch einen „paradoxen Rebound" und führt in eine gegensätzliche Richtung als trainiert. Zum Beispiel zeigen Patienten mit einer posttraumatischen Belastungsstörung übermäßig reduzierte $\operatorname{Alpha}(\alpha)$-Aktivität. Reguliert man die $\alpha$-Aktivität durch NF weiter herunter, kommt es direkt nach dem NF zur sofortigen Erhöhung in Richtung normalisierte $\alpha$-Aktivität (Jokić-Begić und Begić 2003). (iii) Da strukturelle neuronale Verbindungen von der Aktivität des Netzwerks abhängen, kann NF auch zu plastischen Veränderungen der grauen und weißen neuronalen Masse führen (Ros et al. 2014). Werden neuroplastische Mechanismen ausgelöst, können theoretisch Langzeiteffekte erwartet werden.

\section{Neurofeedback als evidenz- basiertes Verfahren in der Therapie}

Die Wirksamkeit von NF wird derzeit im Bereich der hyperkinetischen Störungen (HKS) kritisch untersucht. In einer Metaanalyse der entsprechenden europäischen Arbeitsgemeinschaft wurden verschiedene NF-Protokolle im Vergleich zu aktiven Kontrollgruppen auf die Reduzierung der Symptomatik bei HKS untersucht (Cortese et al. 2016). Effekte auf Hyperaktivität- und ImpulsivitätsSymptomatik wurden über alle verschie- 
denen NF-Protokolle im Vergleich zu unterschiedliche Kontrollgruppen (inbegriffen waren Standardbehandlungen, Wartelisten-Kontrollgruppen, Placebo Neurofeedback bei dem Probanden nur scheinbares Feedback über die eigene Gehirnaktivität erhalten und aktive Kontrollgruppen) untersucht. Es ergaben sich signifikante Ergebnisse nur auf nicht-verblindet erhobene Erfolgsmaße, aber nicht auf verblindet erhobene Erfolgsmaße.

Allerdings müsste, methodisch streng genommen, die Wirksamkeit für verschiedene NF-Protokolle gesondert berechnet werden, wie auch in der Pharmakologie die Wirksamkeit unterschiedlicher Wirkstoffe getrennt nachgeprüft wird. Wird dies missachtet, kann es zu verzerrten Einschätzungen und falschen Schlussfolgerungen kommen. Diese gesonderte Betrachtung ist gegenwärtig durch die geringe Anzahl methodisch robuster Studien (noch) nicht möglich.

Es liegen allerdings Metaanalysen vor, die sich zumindest auf 3 Standardprotokolle, das Theta $(\theta) / \operatorname{Beta}(\beta)$-Ratio, sensorisch motorischer Rhythmus (SMR) und das Training langsamer kortikaler Potenziale (LP), beschränken. Schätzt man die kombinierte Wirksamkeit für diese 3 Protokolle, zeigen sich signifikante Ergebnisse auf verblindet erhobene Erfolgsmaße im Vergleich zu (semi)aktiven Kontrollgruppen (z. B. Micoulaud-Franchi et al. 2014; Cortese et al. 2016). Obwohl nach derzeitigem Kenntnisstand bislang nicht zu beurteilen ist, wie wirksam die einzelnen Standardprotokolle für sich genommen sind, werden diese Protokolle als evidenzbasierte Behandlungsmethoden im Bereich HKS angesehen (• Tab. 1). Eine jüngste Metaanalyse überprüfte zudem langfristige Effekte dieser Standardprotokolle nach 6 Monaten im Vergleich zu einer Gruppe mit herkömmlicher Standardbehandlung (Methylphenidat oder Selbstmanagement) und einer nichtaktiven Kontrollgruppe, zu der alle anderen Bedingungen gezählt wurden (van Doren et al. 2018). Die herkömmliche Standardbehandlung führte direkt nach Abschluss zu stärkeren Effekten auf Unaufmerksamkeit als NF. Im Vergleich zum Behandlungsabschluss erfolgten nach $6 \mathrm{Mo-}$ naten keine weiteren signifikanten Effek-

Psychotherapeut 2019·64:186-193 https://doi.org/10.1007/s00278-019-0351-3

(c) Der/die Autor(en) 2019

\section{S. Enriquez-Geppert}

\section{Neurofeedback aus der Perspektive der Neurowissenschaften. Aktuelle Entwicklungen und Trends}

\section{Zusammenfassung}

Im vorliegenden Beitrag wird dem Leser ein Einblick in den aktuellen Stand der klinischen Neurofeedback-Interventionen sowie der Forschung zum Neurofeedback (NF) gegeben. (i) Die Einführung umfasst die Methode, Wirkungsmechanismen und derzeitige Einsatzbereiche. (ii) Es folgt die Darstellung von NF als evidenzbasiertes Verfahren in der Therapie von psychischen Störungen. Hierbei lassen sich v. a. durch 3 Protokolle bei hyperkinetischen Störungen (HKS) gute Wirkungsergebnisse erzielen. Zudem werden Herausforderungen in der Wirksamkeitsforschung erwähnt. (iii) Ein weiterer Schwerpunkt ist die Beschreibung von neurowissenschaftlich inspirierten NF-Protokollen, die in den letzten Jahren, angeregt durch den aktuellen Kenntnisstand der Neurobiologie und Biopsychologie, entwickelt wurden und als potenzielle therapeutische Interventionen gelten. Als Beispiel wird das Protokoll zur Hochregulierung der frontomedialen Theta-Oszillationen zur Verbesserung exekutiver Funktionen dargestellt. Da diese für eine erfolgreiche Funktionsfähigkeit im Alltag eine Rolle spielen sowie andererseits exekutive Dysfunktionen mit klinisch psychologischen Befunden und reduzierter Lebensqualität in Verbindung stehen, birgt die Untersuchung dieses Protokolls großes Potenzial für die klinische Anwendung. (iv) Weiterhin werden die wichtigsten Schritte und Überlegungen für die Durchführung einer klinischen NF-Studie dargestellt sowie abschließend (v) laufende Studien und neue Entwicklungen, wie die aktuell erstellte Checkliste und Richtlinien zu $\mathrm{NF}$, beschrieben.

\section{Schlüsselwörter}

Neuromodulation - Neurowissenschaftliche klinische Intervention · Hyperkinetisches Syndrom · Konditionierung von Gehirnaktivität . Selbstregulation von Gehirnaktivität

\section{Neurofeedback from the perspective of neurosciences. Current developments and trends}

\section{Abstract}

This article provides the reader with insights into the current state of the clinical application of neurofeedback (NF) interventions as well as into the current research of NF. (i) The introduction gives a clear description of the methods, the mechanisms of action and the current domains of application. (ii) The following section assesses NF as an evidencebased procedure in the therapy of mental disorders. In this respect especially three NF protocols in the domain of attention-deficit hyperactivity disorders show good evidence of efficacy. (iii) Further emphasis is placed on the description of neuroscientifically inspired NF protocols as potential therapeutic tools that arose in recent years following research progress in neurobiology and biopsychology. As an example of such a protocol, the upregulation of frontomidline theta $(\theta)$ oscillations for the improvement of executive functions is given. Since executive functions play a crucial role in successful everyday functioning and executive dysfunctions are associated with a range of psychological disorders and reduced quality of life, this protocol has a great potential for clinical application. (iv) A further section describes important steps and considerations for the implementation of clinical NF studies and finally, (v) current studies and new developments, such as the currently available checklist and guidelines on NF are presented.

\section{Keywords}

Neuromodulation - Neuroscientific clinical interventions - Hyperkinetic syndrome . Conditioning of brain activity - Self-regulation of brain activity 


\section{Infobox 2 Neurofeedback in der praktischen Anwendung \\ Die wichtigste Fachgesellschaft für Neurofeedback (NF) ist die Deutsche Gesellschaft für Biofeedback e.V. (DGBFB, https://www.dgbfb.de/index.php/de): \\ - Die DGBFB bietet Ärzten oder psy- chologischen Psychotherapeuten eine Zusatzausbildung zum "Neurofeedback- Therapeut DGBFB" und für Personen mit Abschlüssen des Gesundheitswesens zum "Neurofeedback-Trainer" an \\ - Die DGBFB veröffentlicht Empfehlungen zur Qualitätssicherung (https://www. dgbfb.de/index.php/de/stellungnahmen/ 144-empfehlungennfb) und Ausbildungs- anforderungen (https://www.dgbfb.de/ index.php/de/ausbildung/46-ausbildung) \\ Finanzierung der NF-Therapie: \\ - Neurofeedback wird durch einen psychologischen Psychotherapeuten mit Kassenzulassung als Bestandteil der von der gesetzlichen Krankenkasse finanzierten Verhaltenstherapie gerechnet \\ - Andernfalls übernehmen die gesetzlichen Krankenkassen die Kosten einer NF-Be- handlung nur in Ausnahmen oder auf ärztliches Rezept in der Ergotherapie. Die Abrechnung erfolgt als individuelle Ge- sundheitsleistung (,IGeL") und wird daher als privatärztliche Leistung abgerechnet}

te. Bezüglich Hyperaktivität und Impulsivität zeigte NF die gleichwertige Wirkung zur herkömmlichen Standardbehandlung direkt nach Behandlungsabschluss. Nach 6 Monaten ergab sich zudem ein nennenswerter Trend zugunsten stabiler Effekte von NF gegenüber beiden Kontrollgruppen, was bezüglich dieser Symptome für einen nachhaltigen Behandlungseffekt sprechen könnte.

Zum Gebiet der pharmakoresistenten Epilepsie liegt ferner eine Metaanalyse vor, die die Wirkung von NF auf die Reduktion der Anfallsfrequenz belegt (SMR und LP; Tan et al. 2009).

Gegenwärtig werden Schlussfolgerungen zur Wirksamkeit durch methodische Schwächen wie Fehlen von adäquaten Kontrollgruppen, Randomisierung und angemessenen Stichprobengrößen erschwert. Dies wird anhand einer Metaanalyse bei psychischen Störungen deutlich (Begemann et al. 2016). Den Aufnahmekriterien entsprachen bei Autismus-Spektrum-Störungen 5, bei Zwangsstörungen 3, bei generalisierter

\section{Infobox 3 Beispiele neuro- wissenschaftlich inspirierter NF-Protokolle}

- Hohes a-Band-Training und Arbeitsgedächtnis (z. B. Zoefel et al. 2011, Escolano et al. 2011)

- a-Lateralisierung und verdeckte Aufmerksamkeit (Okazaki et al. 2015)

- Sensorisch motorischer Rhythmus und Motorik (McFarland et al. 2015)

- Frontomediales $\theta$ und exekutive Funktionen (Enriquez-Geppert et al. 2014)

Angststörung 2 und bei Depression nur eine Studie. Die Verwendung adäquater Kontrollgruppen spielt besonders in Bezug auf die Einschätzung der Ursachen zum Therapieerfolg eine wichtige Rolle (•Abb. 3). Bezüglich NF sind 5 zu unterscheiden: (i) spezifische neurophysiologische Effekte, die durch das Training eines bestimmten Gehirnsignals entstehen, (ii) nichtspezifische Effekte, die NF-kontextspezifisch sind (z.B. Therapeut-Patient-Interaktion in einem neurotechnologischen Kontext), (iii) nichtspezifische Effekte, die generell bei Interventionen entstehen (z. B. Teilnahme an einer Form von kognitivem Training, psychosoziale Effekte, Placebo-Mechanismen usw.), (iv) Wiederholungseffekte und (v) natürliche Effekte, die positiv (z. B. Spontanremission), aber auch negativ (z.B. kognitive Verminderung durch Alterung) ausfallen können (Ros et al. im Druck). Derzeit wird eine wissenschaftliche Debatte über die Größe der verschiedenen Anteile dieser Effekte geführt. Einschätzungen reichen von „ausschließlich Placebo getrieben“ $\mathrm{zu}$ „V.a. NF-spezifisch begründet“" (Thibault et al. 2017; Fovet et al. 2017).

\section{Neurowissenschaftlich inspirierte NF-Protokolle}

Die neurowissenschaftliche Forschung hat in den letzten Jahren zu neuen NFProtokollen (•Infobox 3) geführt, die sich eher auf neurobiologische und verhaltensbezogene Komponenten als auf komplexe Symptome psychischer Störungen konzentrieren. Exemplarisch ist die Forschung zu neuronalen Oszillationen.

\section{Infobox 4 Exekutive Funktionen}

Exekutive Funktionen sind ein Sammelbegriff für zusammenhängende höhere kognitive Funktionen, die primäre kognitive Funktionen wie z. B. Aufmerksamkeit und Gedächtnis kontrollieren, um flexibles, zielgerichtetes Verhalten und Denken in einer komplexen Umwelt zu ermöglichen. Exekutive Funktionen sind für die erfolgreiche Funktionsfähigkeit im Alltag bedeutsam und stehen beispielsweise im Zusammenhang mit:

- Lernprozessen

- sozialen Fähigkeiten

- akademischer und beruflicher Leistung

Verminderte exekutive Funktionsleistung hängen mit psychischen Störungen zusammen, beispielsweise mit:

- depressiven Episoden

- Angststörungen

- leichten kognitiven Störungen

- hyperkinetischen Störungen

- primärem Parkinson-Syndrom

Schizophrenie

Merke. Als neuronale Oszillationen wird die rhythmische neuronale Aktivität bezeichnet, die sich in verschiedenen Frequenzen $(\delta, \theta, \alpha, \beta, \gamma)$ im EEG zeigt. Neuronale Oszillationen kommen auf unterschiedlichen neuronalen Organisationsstrukturen vor, z. B. bei Membranpotenzialen, neuronalem Feuern einzelner Neurone, aber auch bei synchronisierten neuronalen Netzwerken.

Neuronale Oszillationen wirken als Koordinationsmechanismus innerhalb und zwischen Gehirnarealen und sind funktional relevant für Kognition (Herrmann und Knight 2001). So sind etwa die erhöhte Aktivität im oberen $\alpha$-Band mit guter Leistung in mentaler Rotation und $\gamma$-Oszillationen mit Merkmalsintegration, neuronalen Bindungsprozessen und bewusster Wahrnehmung assoziiert (Engel und Singer 2001). Die Zusammenhänge zwischen neuronalen Aspekten von Kognition und Verhalten sind Ansatzpunkte für die Forschung und dienen als Basis für die Entwicklung neuer NF-Protokolle, die somit einen direkten neuromodulatorischen Zugang ermöglicht.

Ein kognitiv-oszillatorischer Zusammenhang besteht auch für exekutive Funktionen (-Infobox 4), die von einem breit verteilten neuronalen, sog. 


\begin{tabular}{|c|c|c|c|}
\hline Standardprotokolle & Charakteristiken & $\begin{array}{l}\text { Anzahl }(n) \text { der } \\
\text { Sitzungen }\end{array}$ & $\begin{array}{l}\text { Elektroden- } \\
\text { position }\end{array}$ \\
\hline \multirow[t]{2}{*}{$\theta / \beta$-Ratio (TBR) } & $\begin{array}{l}\text { Frequenzbandtraining } \\
(4-7 \mathrm{~Hz} / 12-21 \mathrm{~Hz})\end{array}$ & \multirow[t]{2}{*}{ Ca. $30-40$} & \multirow[t]{2}{*}{ Fz oder Cz } \\
\hline & $\begin{array}{l}\text { Zielt auf abnormal hohes } \\
\theta / \beta \text {-Ratio, hohes } \theta \text { und/ } \\
\text { oder zu niedrig ausgeprägte } \\
\beta \text {-Aktivität }\end{array}$ & & \\
\hline $\begin{array}{l}\text { Sensorisch motorischer } \\
\text { Rhythmus (SMR) }\end{array}$ & $\begin{array}{l}\text { Frequenzbandtraining } \\
(10-14 \mathrm{~Hz})\end{array}$ & - & C3, Cz, oder C4 \\
\hline $\begin{array}{l}\text { Langsame kortikale } \\
\text { Potenziale (LP) }\end{array}$ & $\begin{array}{l}\text { Steigerung und Verrin- } \\
\text { gerung des neuronalen } \\
\text { Aktivierungsniveaus }\end{array}$ & Ca. 35 & $\mathrm{Cz}$ \\
\hline
\end{tabular}

frontozingulär-parietalen Netzwerk implementiert werden (Niendam et al. 2012). Forschungsergebnisse unterstreichen die Relevanz von $\theta$-Oszillationen $(4-8 \mathrm{~Hz})$ für die Kommunikation innerhalb dieser Gehirnareale und des gesamten Netzwerks (Sauseng et al. 2007). Während gesteigerter kognitiver Verarbeitung durch exekutive Funktionen wird eine erhöhte $\theta$-Aktivität beobachtet, die im EEG typischerweise an frontomedialen ( $\mathrm{fm}$ ) Elektrodenpositionen gemessen wird (Cavanagh und Frank 2014).

Merke. Das fm $\theta$ gilt als neuronale Arbeitssprache von exekutiven Funktionen (Cavanagh und Frank 2014).

Implikationen für die klinische Erforschung resultieren aus folgenden Ergebnissen. Exekutive Dysfunktionen (i) finden sich in einer Reihe von psychischen Störungsbildern wieder, (ii) stehen im Zusammenhang mit einem Funktionsverlust im Alltag und einer Beeinträchtigung der Lebensqualität (Stern et al. 2016; Nguyen et al. 2019) und (iii) gehen mit veränderter $\theta$-Aktivität einher.

Für die klinische Anwendung ist ausschlaggebend, ob exekutive Funktionen durch fm- $\theta$-NF verbessert werden können. Diese Fragestellung wurde bereits in unterschiedlichen Laboren untersucht (Wang und Hsie 2014; Enriquez-Geppert et al. 2014). In den Studien der eigenen Arbeitsgruppe wurde ein individualisiertes Protokoll entwickelt, das die $\theta$-Aktivität hochtrainiert und mit einem Pseudo-NF als aktive Kontrollgruppe vergleicht. Die Individualisierung betrifft den genauen Frequenzbeund basiert auf der probandenspezifischen maximalen $\theta$-Frequenz. Bezüglich der Operationalisierung des Pseudo-NF wurde in eigenen Studien das Feedback eines Probanden der Experimentalgruppe aufgenommen und einem anderen der aktiven Kontrollgruppe gegeben. Des Weiteren wurden exekutive Funktionen anhand von 4 Komponenten (Handlungsüberwachung, Inhibition, Aktualisierung des Arbeitsgedächtnisses und dem Aufgabenwechsel) vor und nach dem NF-Training untersucht. Die Ergebnisse zeigen (i) im Verlauf des Trainings eine signifikant stärkere Erhöhung der $\theta$-Aktivität in der NFGruppe im Vergleich zum Pseudo-NF. Der Vergleich der Prä-post-Messungen ergab auch hier signifikante Transfereffekte, (ii) eine verbesserte Performanz (iii) eine erhöhte fm- $\theta$-Aktivität während der Aufgabendurchführung in der NF-Gruppe im Vergleich zur PseudoNF-Gruppe (• Abb. 4).

\section{Evidenzbasierte Neurofeed- back-Forschung}

Da die NF-Studienplanung und -Studiendurchführung allgemein Besonderheiten beinhalten, werden diese im folgenden Abschnitt hervorgehoben und ein erster Einblick gegeben. Insgesamt sind bei der Vorbereitung von NF-Studien 4 Domänen zu berücksichtigen (ausführliche Beschreibung: Enriquez-Geppert et al. 2017; - Abb. 5). reich, der für das NF verwendet wird, der Aktualisierung des Arbeitsgedächtnisses und des Aufgabenwechsels sowie

\section{Infobox 5 Empfohlene Literatur II}

- Enriquez-Geppert S, Huster RJ, Herrmann CS (2017) EEG-neurofeedback as a tool to modulate cognition and behavior: a review tutorial. Front Hum Neurosci 11:51

- Ros T, Baars B, Lanius RA, Vuilleumier P (2014) Tuning pathological brain oscillations with neurofeedback: a systems neuroscience framework. Front Hum Neurosci 8:1008

Vorbereitung und Durchführung klinischer NF-Studien erfolgen auf Basis randomisierter kontrollierter Studiendesigns mit den folgenden 3 Merkmalen: (i) Verwendung von Kontrollgruppen (mit zumindest einer aktiven Kontrollgruppe oder einer Kontrollbedingung), (ii) randomisierte Zuteilung der Probanden zu den Gruppen und (iii) Verblindung (der Patienten und der Untersucher).

Bezüglich der spezifischen NF-Charakteristiken sind 4 Aspekte zu beachten. (i) Zunächst geht es um die Festlegung der benötigen Sitzungsanzahl, bei der sich Untersucher an vergleichbaren Studien oder Erfahrungswerten orientieren. Allerdings kann auch bis zu einem Kriterium trainiert werden, wie z. B. bis zu einer bestimmten Symptomlinderung. (ii) Weiterhin ist die Verteilung der einzelnen Sitzungen über die gesamte Trainingsperiode wichtig, zu der es aber noch keine systematischen Untersuchungen gibt. Beispiele reichen von 2-mal/Tag bis einmal/Woche. (iii) Der dritte Aspekt bezieht sich auf die Sitzungsdauer und das Einbringen von Pausen. (iv) Zudem stellt die Anleitung, wie ein Gehirnsignal verändert werden kann, einen wichtigen Gesichtspunkt dar und variiert von konkreten bis hin zur gänzlichen Vermeidung von Instruktionen. Im Fall des Wiedererlangens motorischer Fähigkeiten bei Patienten mit intrazerebraler Blutung werden die Patienten instruiert, sich bestimmte motorische Bewegungen vorzustellen, da bekannt ist, dass offene und vorgestellte Bewegungen auf zumindest ähnliche neuronale Ressourcen zurückgreifen. Bei einem $\theta$ - $\beta$-Ratio-Training 


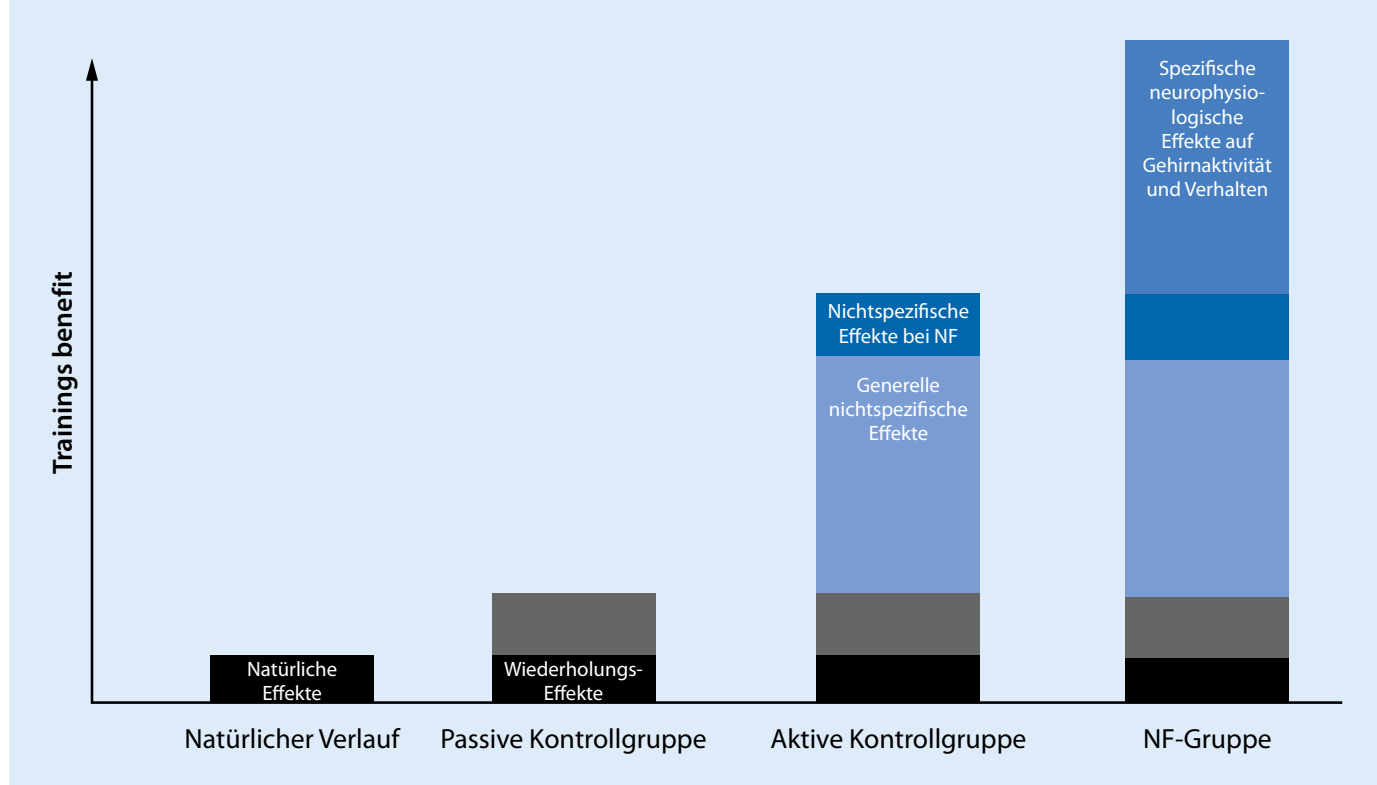

Abb. $3<$ Hypothetisches Modell der multiplen Effekte bei Neurofeedback (NF)

5 NF Elektroden, 2 EOG, Referenz, Erdungselektrode

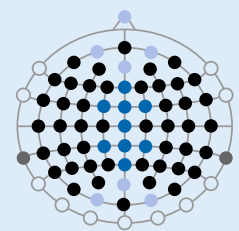

Veränderungen der Theta-Amplitude über das NF

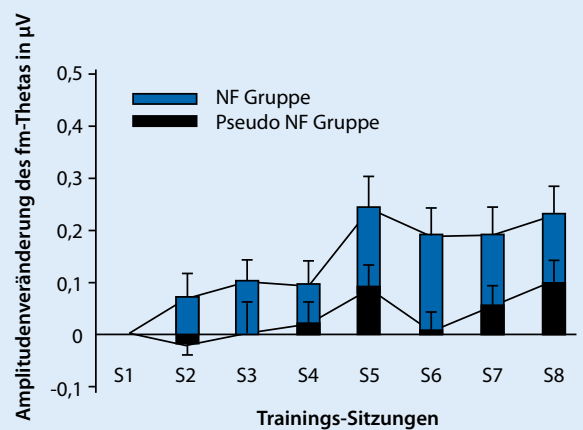

Transfereffekte des Trainings auf vier exekutive Funktionen und ereigniskorreliertes Theta

\begin{tabular}{|l|l|}
\hline $\begin{array}{l}\text { Aufgaben zu exekutiven } \\
\text { Funktionen }\end{array}$ & Prä-Post Trainingsunterschiede \\
\hline STROOP & $\ldots$ keine Gruppenunterschiede \\
\hline STOP-SIGNAL & $\ldots$ keine Gruppenunterschiede \\
\hline DREI-ZURÜCK & $\begin{array}{l}\text { NF vs. Pseudo-NF Gruppe: } \\
\text { Signifikant stärkere Verbesserung der } \\
\text { Genauigkeit in der NF-Gruppe im } \\
\text { Vergleich zur aktiven Kontrollgruppe }\end{array}$ \\
\hline AUFGABENWECHSEL & $\begin{array}{l}\text { NF vs. Pseudo-NF Gruppe: } \\
\text { Signifikant stärker reduzierte } \\
\text { Reaktionszeiten in der NF-Gruppe im } \\
\text { Vergleich zur aktiven Kontrollgruppe }\end{array}$ \\
\hline AUFGABENBEZOGENES FM-THETA & $\begin{array}{l}\text { NF vs. Pseudo-NF Gruppe: } \\
\text { Generell erhöhte fm-Theta Aktivität } \\
\text { über die Aufgaben hinweg in der NF- } \\
\text { Gruppe und reduzierte fm-Theta } \\
\text { Aktivität bei der Pseudo-NF Gruppe }\end{array}$ \\
\hline
\end{tabular}

Abb. 4 \ Studienergebnisse des frontomedialen Theta-Protokolls. EOG Elektrookulogramm, NF Neurofeedback

werden Probanden oft instruiert, sich lediglich zu entspannen, aber gleichzeitig aufmerksam zu sein. Bei wiederum anderen NF-Protokollen werden nur relativ grobe Instruktionen gegeben (einfach etwas ausprobieren, mentale Rotation von bekannten Gegenständen, mentales Navigieren durch bekannte Gebäude etc.). Generell werden interindividuelle Unterschiede bei der Verwendung von mentalen Strategien beobachtet und noch keine allgemein bewährten Strategien für die Selbstregulation identifiziert (- Infobox 5).

Die Merkmalsextraktion betrifft die (i) Auswahl des zu trainierenden neuronalen Merkmals und (ii) die Anzahl und Position der Elektroden. Die Entscheidung für ein bestimmtes zu trainierendes neuronales Merkmal sowie die Frage, wie dieses Merkmal am besten gemessen und trainiert werden kann, sollte sich am aktuellsten Erkenntnisstand ausrichten. $\mathrm{Zu}$ beachten ist, dass interindividuelle Unterschiede der Gehirnanatomie und -funktion zu topografischen Unterschieden der EEG-Aktivität führen und zur Kompensation die Verwendung mehrerer Elektroden hilfreich ist. Ein weiterer 


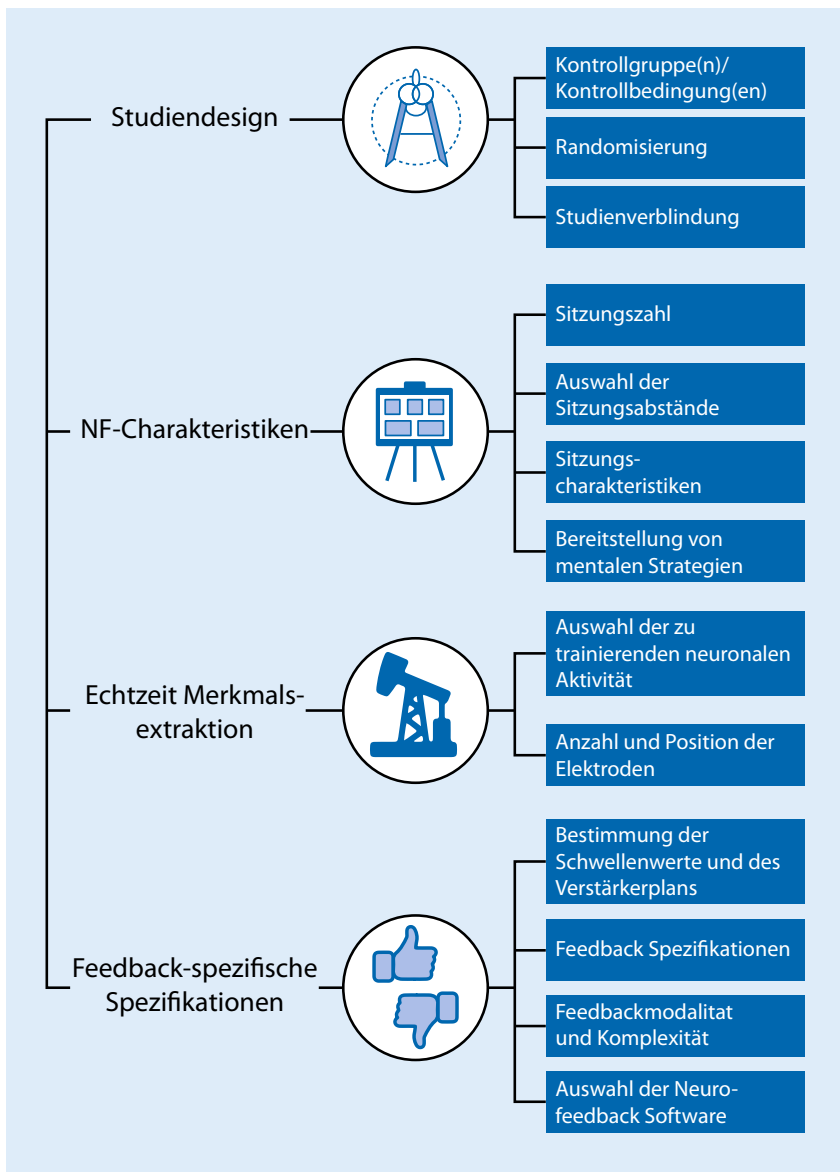

Speicherung der Forschungsdaten beschreibt. Die CRED-NF-Checkliste soll einerseits dazu beitragen, Untersucher bei qualitätssichernden Maßnahmen zu unterstützen und andererseits die Einschätzung einzelner Wirkmechanismen zu verbessern.

Des Weiteren ist auf die sog. International Collaborative ADHD Neurofeedback Study hinzuweisen, die als große doppelblinde Multizenterstudie durchgeführt wird (Arnold et al. 2013). Als eine der Besonderheiten dieser Studie ist hervorzuheben, dass sowohl NF-Skeptiker als auch NF-Forscher beteiligt sind.

Das fm- $\theta$-Protokoll ist derzeit als therapeutische Intervention aktueller Forschungsgegenstand in subklinischen und klinischen Gruppen an mehreren Universitäten.

Abb. $5 \triangleleft$ Entscheidungsbaum zum Erstellen einer Neurofeedback-Studie
Vorteil ist das verbesserte Signal-RauschVerhältnis.

Betreffend feedbackbezogener Spezifikationen geht es um die (i) Festlegung einer kritischen Schwelle, die die Gehirnaktivität für ein bestimmtes Feedback-Signal überschreiten muss, und um die Bestimmung des genauen Verstärkerplans. Das Feedback-Signal indiziert die relative Veränderung der Gehirnaktivität zur Ruhemessung vor der jeweiligen aktuellen Sitzung, der Durchschnittsaktivität einer vorherigen Sitzung oder im Vergleich zu normativen Daten. (ii) Bei der genauen Gestaltung des Feedbacks spielen lerntheoretische Überlegungen eine Rolle und betreffen z. B. die Auswahl eines kontinuierlichen oder festen Quotenplans. (iii) Die Entscheidung, welche Feedback-Modalität (auditiv, taktil, visuell etc.) und Komplexität (einfache Figuren, bewegte Bilder etc.) gewählt werden, beruht derzeit auf praktischen Erfahrungen und patientenspezifischen Charakteristiken oder ist durch die Verwendung einer spezifischen NF-Software vorgege- ben. (iv) Letztendlich hat die Wahl der NF-Software einen bedeutsamen Einfluss auf die Wahlfreiheit der genannten Spezifikationen.

\section{Neue Impulse, zukunftsweisen- de Entwicklungen und laufende Studien}

Derzeitig werden Konsensusrichtlinien und Checklisten für experimentelle Designs und des Berichtens von Forschungsergebnissen entwickelt, die auf methodische Genauigkeit abzielen (Ros et al. im Druck, Vordruck abrufbar unter: https://psyarxiv.com/nyx84). Dabei haben führende NF-Wissenschaftler eine sog. CRED-NF(Consensus on the reporting and experimental design of clinical and cognitive-behavioural neurofeedback studies)-Checkliste ausgearbeitet, die vorexperimentelle Schritte wie die Vorregistrierung einer Studie, die Spezifikation von Kontrollgruppen, weiteren Kontrollmaßen, Feedbackspezifikationen, Ergebnismaßen und der

\section{Fazit für die Praxis}

- Zur Behandlung der hyperkinetischen Störungen (HKS) existieren 3 evidenzbasierte Neurofeedback(NF)Protokolle.

- Materialien zur Planung von NFStudien und zur Einschätzung der Studienqualität stehen zur Verfügung.

- Das Potenzial neurowissenschaftlich inspirierter NF-Protokolle liegt in der verbesserten Konstruktvalidität als Voraussetzung für die Behandlungswirksamkeit.

\section{Korrespondenzadresse}

Assistant Professor Dr. Stefanie EnriquezGeppert

Abteilung für Klinische und EntwicklungsNeuropsychologie, Universität Groningen Grote Kruisstraat 2/1, 9712 TS Groningen, Niederlande

s.enriquez.geppert@rug.nl

Danksagung. Die Autorin dankt JEG, SH und JGE für das kritische Lesen des Manuskripts und den Gutachtern für das konstruktive Feedback.

\section{Einhaltung ethischer Richtlinien}

Interessenkonflikt. S. Enriquez-Geppert gibt an, dass kein Interessenkonflikt besteht. 
Für diesen Beitrag wurden von der Autorin keine Studien an Menschen oder Tieren durchgeführt. Für die aufgeführten Studien gelten die jeweils dort angegebenen ethischen Richtlinien

Open Access. Dieser Artikel wird unter der Creative Commons Namensnennung 4.0 International Lizenz (http://creativecommons.org/licenses/by/4.0/deed de) veröffentlicht, welche die Nutzung, Vervielfältigung, Bearbeitung, Verbreitung und Wiedergabe in jeglichem Medium und Format erlaubt, sofern Sie den/die ursprünglichen Autor(en) und die Quelle ordnungsgemäßnennen, einen Linkzur Creative Commons Lizenz beifügen und angeben, ob Änderungen vorgenommen wurden.

\section{Literatur}

Arnold LE, Lofthouse N, Hersch S, Pan X, Hurt E, Bates B (2013) EEG neurofeedback for ADHD: double-blind sham-controlled randomized pilot feasibility trial. J Atten Disord 17(5):410-9. https://doi.org/10.1177/1087054712446173

Begemann MJH, Florisse EJR, van Lutterveld R, Kooyman M, Sommer IE (2016) Efficacy of EEG neurofeedback in psychiatry: A comprehensive overview and meta-analysis. Translational Brain Rhythmicity 1(1):19-29

Cavanagh JF, Frank MJ (2014) Frontal theta as a mechanism for cognitive control. Trends Cogn Sci 18:414-421

Cortese S, Ferrin M, Brandeis D, Holtmann M, Aggensteiner P, Daley D et al (2016) Neurofeedback for attention-deficit/hyperactivity disorder: MetaAnalysis of clinical and neuropsychological outcomes from randomized controlled trials. J Am Acad Child Adolesc Psychiatry 55(6):444-455

van Doren J, Arns M, Heinrich H, Vollebregt MA, Strehl U, Loo SK (2018) Sustained effects of neurofeedback in ADHD: a systematic review and meta-analysis. Eur Child Adolesc Psychiatry 28(3):293-305

Engel AK, Singer W (2001) Temporal binding and the neural correlates of awareness. Trends Cogn Sci 5(1):16-25. https://doi.org/10.1016/S13646613(00)01568-0

Enriquez-Geppert S, Huster RJ, Figge C, Herrmann CS (2014) Self-regulation of frontal-midline theta facilitates memory updating and mental set shifting. Front Behav Neurosci 8:420

Enriquez-Geppert S, Huster RJ, Herrmann CS (2017) EEG-neurofeedback as a tool to modulate cognition and behavior: a review tutorial. Front Hum Neurosci 11:51

Escolano C, Aquilar M, Minguey J (2011) EEG-based upper alpha neurofeedback training improves working memory performance. Conf Proc IEEE Engg Med Biol Soc, S 2327-2330

Fovet T, Micoulaud-Franchi JA, Vialatte FB, Lotte F, Daudet C, Batail JM, Ros T et al (2017) On assessing neurofeedback effects: should doubleblind replace neurophysiological mechanisms? Brain 140(10). https://doi.org/10.1093/brain/ awx211

Herrmann CS, Knight RT (2001) Mechanisms of human attention: event-related potentials and oscillations. Neurosci Biobehav Rev 25:465-476

Jokic-Begic N, Begic D (2003) Quantitative electroencephalogram ( $\mathrm{qEEG}$ ) in combat veterans with post-traumatic stress disorder (PTSD). Nord Psychiatry 57:351-355
Lacroix JM (1986) Mechanisms of biofeedback control: on the importance of verbal (conscious) processing. In Davidson RJ, Schwartz GE, Shapiro D (Hrsg) Consciousness and Self-Regulation. New York: Plenum, S 137-162. https://doi.org/ 10.1007/978-1-4757-0629-1_6

McFarland DJ, Sarnacki WA, Wolpaw JR(2015) Effects of training pre-movementsensorimotor Rythms on behavioral performance: a pilot study. J Neural Eng 12(6). https://doi.org/10.1088/1741-2560/ $12 / 6 / 06602$

Micoulaud-Franchi JA, Geoffroy PA, Fond G, Lopez R Bioulac S, Philip P (2014) EEG neurofeedback treatments in children with ADHD: an updated meta-analysis of randomized controlled trials. Front Hum Neurosci 8:906

Nguyen CM, Copeland CT, Lowe DA, Heyanka DJ, Linck JF (2019) Contribution of executive functioning to instrumental activities of daily living in older adults. Appl Neuropsychol Adult 15:1-18

Niendam TA, Lair AR, Kimberly LR, Dean YM, Carter CS (2012) Meta-analytic evidence for a superordinate cognitive control network subserving diverse executive functions. Cogn Affect Behav Neurosci 12(2):241-268

Okazaki YO, Horschig JM, Luther L, Oostenveld R, Murakami I, Jensen O (2015) Real-time MEG neurofeedback training of posterior alpha activity modulates subsequent visual detection performance. Neuroimage 107:323-332

Ros T, Baars BJ, Lanius RA, Vuilleumier P (2014) Tuning pathological brain oscillations with neurofeedback: a systems neuroscience framework. Front Hum Neurosci 8:1008

Ros T, Enriquez-Geppert S, Young K, Wood G, Sulzer J, Sterman $B$ et al Consensus on the reporting and experimental design of clinical and cognitivebehavioural neurofeedback studies (CREDnf checklist). https://doi.org/10.31234/osf.io/ nyx84

Sauseng P, Hoppe J, Klimesch W, GerloffC, Hummel FC (2007) Dissociation of sustained attention from central executive functions: local activity and interregional connectivity in the theta range. Eur JNeurosci 25:587-593

Stern A, Pollak Y, Bonne O, Malik E, Maeir A (2016) The relationship between executive functions and quality of life in adults with ADHD. J Attent Disorders 21(4):323-330

Tan G, Thornby J, Hammond DC, Strehl U, Canady B, Arnemann K, Kaiser DA (2009) Meta-analzsis of EEG biofeedback in treating epilepsy. Clin EEG Neurosci 40(3):173-9. https://doi.org/10.1177/ 155005940904000310

Thibault RT, Lifshitz M, Raz A (2017) Neurofeedback or neuroplacebo? Brain 140(4):862-864

Wang J-R, Hsieh S (2014) Neurofeedback training improves attention and working memory performance. Clin Neurophysiol 142(1):2406-2420

Wood G, Kober SE, Witte M, Neuper C (2014) On the need to better specify the concept of "control" in brain-computer-interfaces/neurofeedback research. Front Syst Neurosci 8:171. https://doi. org/10.3389/fnsys.2014.00171

Zoefel B, Huster RJ, Herrmann CS (2011) Neurofeedback training of the upper alpha frequency band in EEG improves cognitive performance. Neuroimage 54:1427-1431

\section{Depressionen unter deutschen Ärzten weit verbreitet}

Ausgebrannt? Depressiv? Frustriert? Wie sehr Mediziner in Deutschland gestresst und überfordert sind, ergab eine Online-Befragung des Informationsportals Medscape von 615 Ärzten. Dem neuen Report "Burnout und Depressionen bei Ärzten in Deutschland" zufolge berichtete fast jeder zweite Arzt von Gefühlen körperlicher, emotionaler und mentaler Erschöpfung. Jeder vierte Arzt gab an, unter Depressionen und depressiven Verstimmungen zu leiden. $12 \%$ berichteten über Symptome eines Burnouts, $9 \%$ nannten eine Kombination aus beiden Beschwerden.

\section{Welche Faktoren belasten}

Bei Depressionen liegen die deutschen Ärzte nach den Angaben von Medscape im Ländervergleich an der Spitze. Deutsche Ärzte, die unter einem Burnout leiden, empfinden vor allem die Verwaltungsaufgaben (52\%) als große Belastung. Zu viele Arbeitsstunden (50), mangelnde Anerkennung im sozialen Umfeld (36), zu starke Gewinnorientierung (32), unzureichende Vergütung (26), staatliche Regulierungen (18) oder zunehmende Computerisierung (18) folgen als weitere wichtige Faktoren.

\section{Unbehandelte Burn-outs}

Über die Hälfte (60\%) der befragten Mediziner gaben an, keine professionelle Hilfe gegen ihre Depression/ihren Burnout zu suchen. Die Erkrankung sei nicht schwer genug oder zu wenig Zeit, durch die Arbeit oder die Einschätzung, die Situation auch so bewältigen zu können, lautete die Begründung.

Quelle: Ärzte Zeitung $\hookleftarrow$ basierend auf: Medscape, News 20.2.2019 (www.medscape.com) 